\title{
New Vision Sensor to Measure Gas Pressure
}

\author{
Krzysztof Murawski \\ Institute of Teleinformatics and Automatics, Department of Cybernetics, Military University of Technology, \\ Kaliskiego Str. 2, 00-908, Warsaw, Poland, k.murawski@ita.wat.edu.pl
}

\begin{abstract}
The paper presents the construction and operation of a video sensor developed for video-manometer. In the publication the use of video-manometer for measuring gas pressure is presented. A characteristic feature of the device is pressure measurement based on diaphragm deformation and digital image processing. Presented measuring technique eliminates restrictions in the construction of the measuring apparatus arising from non-linear nature of diaphragm deformation. It also allows performing measurements of gas pressure, also of explosive gas, providing galvanic isolation between the factor measured and the measuring device. The paper presents the results of video-manometer calibration and measurements taken during the laboratory tests. It has been shown that the developed video-manometer, that is equipped with a flat silicone diaphragm, allows measuring the gas pressure in the range of $0-100$ mbar with an error less than $2 \%$. In the experiments the $\mathrm{CO} 2$ pressure was measured.
\end{abstract}

Keywords: Measuring technique, image processing, measurement of pressure, gas sensor, remote sensing.

\section{INTRODUCTION}

$\mathrm{P}^{\mathrm{n}}$ RESSURE SENSORS play an important role in the control of technological processes. They ensure safe operation of devices and also enable operation of autonomous control systems and the surveillance of technical systems. They are found in almost every branch of the industry. They can be found in the food, chemical, fuel, civil and military aviation industry, in medicine [1-5], and in others. They are also used in research and development works [6] as well as in diagnostics. Due to the large area of applications and the range of measured pressures, a significant number of pressure sensors and transducer structures have been developed. The direct measurement of the pressure is performed using: Diaphragm, Bourdon Tube, Strain Gauge Diaphragm, Capacitance Diaphragm, $\mathrm{Hg}$ Manometer, Liquid Manometer, Radioactive Gauge, McLeod compression manometer, U-tube Manometer [7].

The pressure is measured indirectly, when the properties of gases are known, using: Momentum Transfer Viscosity (Quartz Fiber, Rotating), Energy Transfer - Thermal conductivity (Thermocouple, Pirani, Thermistor, Convection Gauge), Charge generation with Hot Cathode (Triode Ion, Bayard - Alpert Ion, Schulz - Phelps Ion, Extractor Ion) and Cold Cathode (Magnatron, Inverted Magnatron, Double Inverted Magnatron) as well as Mass Spectrometer [8]. These techniques cover the pressure measuring range from $1.33 \mathrm{e}-15$ bar to 10 bar [8]. Today, a large emphasis is placed on the construction of intelligent pressure sensors. Such sensors, in addition to the sensing element, include electronic systems required for the linearization of the sensor characteristics, to eliminate the effects of temperature, autocalibration and measurement range and reference point (zero and alarm thresholds) settings. The observed sensors for measuring the gas pressure with the optical path measurement fit the trend. The following solutions have been noted in the literature. The first uses two photodiodes and one IR LED diode [9]. In the considered arrangement, Fig.1.a), the LED diode along with one of the photodiodes forms a slotted optocoupler. The second photodiode produces a reference signal used to stabilize the a)

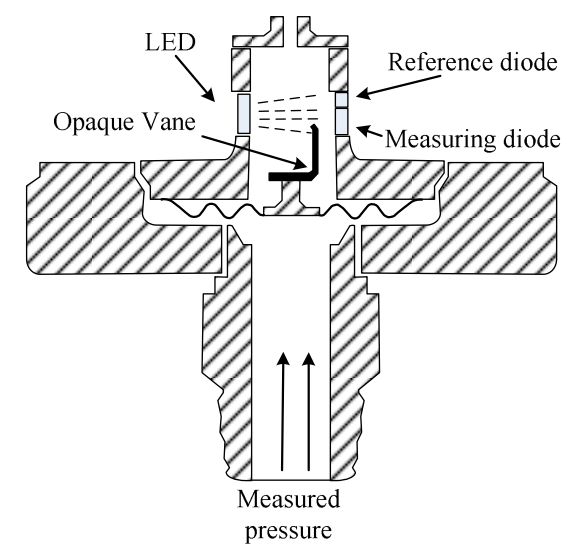

b)

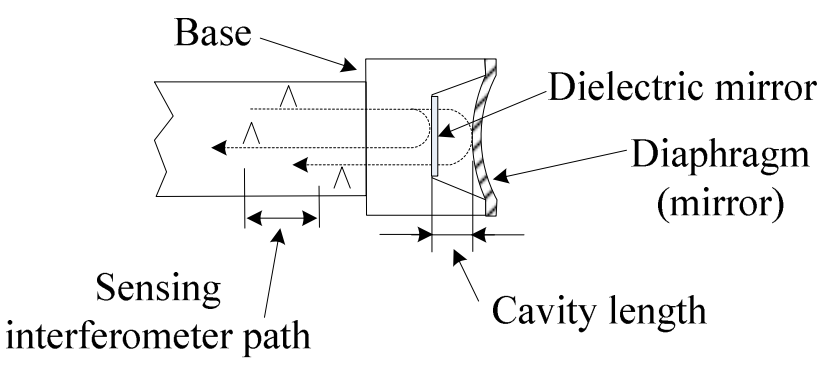

Fig.1. Sensors for measuring pressure with the optical path [5].

sensor operation. An addition to the optical path is the corrugated diaphragm and the shutter (blade). With the increase in the measured pressure, the diaphragm slightly deforms below $0.5 \mathrm{~mm}$ [8], while lifting the blade. The blade blocks the path of light, the intensity of which is measured. Sensors of this type are available for ranges from 0.35 bar to 4.13 bar, assuring a measurement accuracy of about $0.1 \%$. The second solution, Fig.1.b), consists of using an optical fiber with a diaphragm measuring head at the end [10]. The gas pressure is measured using the correlation between the gas pressure applied and the intensity of light reflecting from the surface of the diaphragm [10]. The width 
of the optical cavity located between the diaphragm and the optical fiber is also often measured [11]. This distance is determined using a Fabry-Perot interferometer - its operation is discussed in [12]. The pressure measurement result is presented as a value of DC voltage in a range from $0 \mathrm{~V}$ to $5 \mathrm{~V}$ or $0 \mathrm{~V}$ to $10 \mathrm{~V}$ [11]. The measurement result can also be expressed in the form of a current intensity from $4 \mathrm{~mA}$ to $20 \mathrm{~mA}$. Pressure measurement using fiber optic Bragg grating sensors is also a known solution. The solution presented in [13] allowed carrying out the pressure measurement in the range from 0 bar to 1.8 bar with a measurement error percentage of $3.13 \%$.

\section{MOTIVATION}

The aim of this work was to develop a video-manometer and show a practical application of a new method of measuring pressure, especially gas pressure. The proposed method is based on visual measuring of changes in the width or area of the marker placed on the surface of the deformable diaphragm.

The main advantages of the presented technique are noncontact measurement; galvanic isolation of the sensor from the chamber of increased and/or decreased pressure; high sensitivity, reproducibility and accuracy in carried out measurements; interference resistance; lack of frictional and mechanical components; design simplicity; small and scalable sensor sizes; the ability to measure the pressure of gases using diaphragms: flat, corrugated, bellows and capsulated; low-cost implementation; the possibility of using video fiber optics; real-time visual sensor operation self-diagnostics.

Pressure sensors that use the principles of optics exhibit optimum properties for their use in explosive and medical environments. The proposed sensor design extends the already known properties of optical sensors with the real time vision self-diagnostics function. The developed videomanometer can be used in medicine, for example, or in control systems and gas transportation systems.

\section{IMAGE PROCESSING TECHNIQUE}

The presented image processing technique determines the width of the marker based on a single photo.

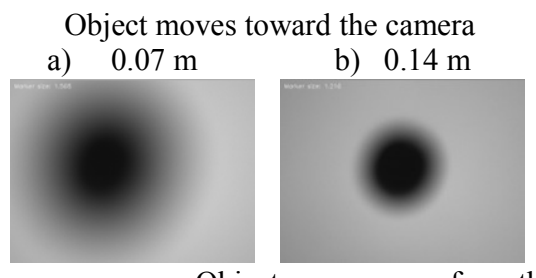

Sharp image

c) $0.23 \mathrm{~m}$

Object
$0.31 \mathrm{~m}$
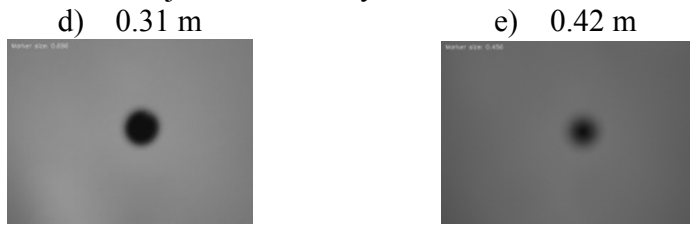

Fig.2. An exemplary sequence of recorded images.
The essence of the presented solution is in the image analysis resulting from the object moving away from the position for which the focus was set, Fig. 2.

Fig.3. shows the profiles of the horizontal lines of the images (Fig.2.a), Fig.2.c), Fig.2.e)) indicated by the center of gravity of the marker as well as their behavior during changes in focus of the image. For the sharp marker view, Fig.2.c), sections AA' and BB' in Fig.3. are almost vertical, which indicates a sharp cut-off of the view of the marker from the background. When the marker gets closer to the camera, the image becomes blurred, Fig.2.a) and Fig.2.b). As a result, points $\mathrm{A}^{\prime}$ and $\mathrm{B}$ ' move in the direction of $\mathrm{A}$ " and B", respectively. At the same time, the distance between points $\mathrm{A}$ and $\mathrm{B}$ remains almost unchanged. When the object with the marker moves away from the camera, points A" and B" return back to their initial position (A" $\rightarrow$ A', B" $\rightarrow$ B'), and the image comes into focus again. Further distancing of the object from the camera results in a repeated blurring of the image and the marker becoming smaller, Fig.2.d) and Fig.2.e). In the case under consideration, the movement of points $\mathrm{A} \rightarrow \mathrm{A}$ "' and $\mathrm{B} \rightarrow$ B"' is observed. The position of points A' and B' remains unchanged.

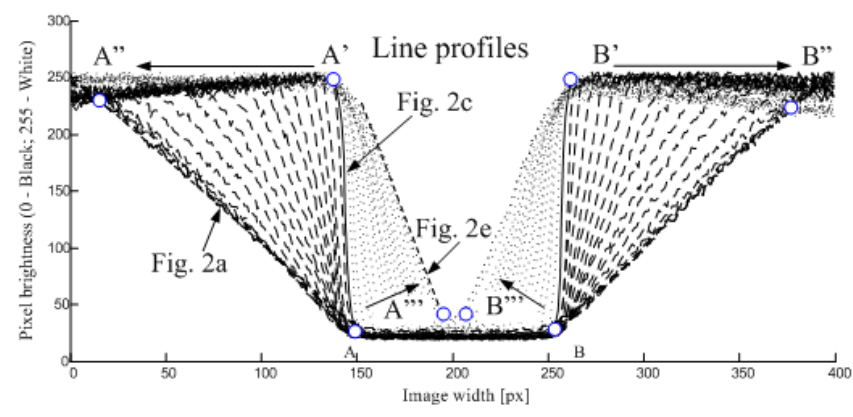

Fig.3. Horizontal line profiles for images from Fig.2.a), Fig.2.c), Fig.2.e) determined by the center of gravity of the object after standardizing brightness.

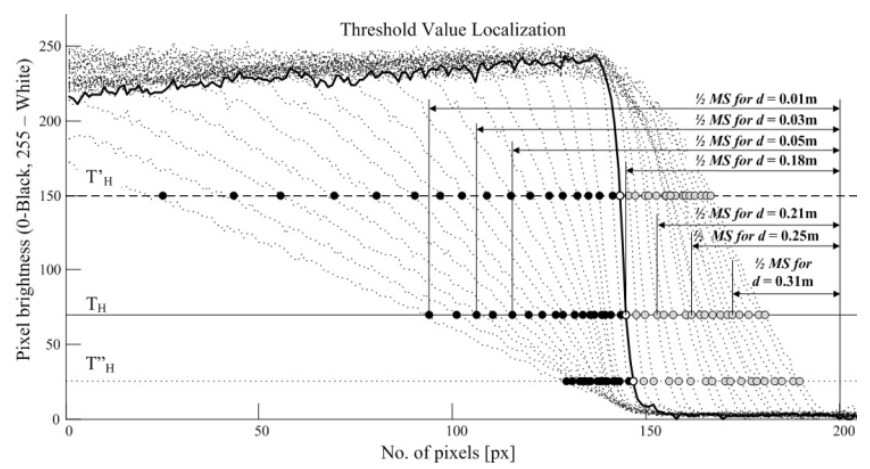

Fig.4. Distribution of essential points due to determining the distance to the object for the accepted threshold value $\mathrm{T}_{\mathrm{H}}$ at $\mathrm{L}_{\mathrm{MIN}}=0.07 \mathrm{~m}, \mathrm{~L}_{\mathrm{MAX}}=0.42 \mathrm{~m}$ and $\Delta \mathrm{L}=0.01 \mathrm{~m}$.

The example sequence of changes in the position of points A, A', B, B' determined during the marker moving toward and away from the camera is presented in Fig.3. and Fig.4. Changing the position of points A, A', B, B' was used to determine the width of the marker. For this purpose, the 
image from the camera underwent defuzzification. Defuzzification was performed using image binarization with a threshold $\mathrm{T}_{\mathrm{H}}$ equal to 70 .

The selection of binarization consists in determining such a $T_{H}$ value to obtain a uniform distribution of points intersecting the profile image horizontal lines (indicated by the center of gravity of the marker) with the line illustrating the tested value $T_{H}$, Fig. 4 . The locations of the determined points are unambiguously associated with the width of the marker MS and its distance $d$ from the camera. The distribution of points is described by the equation in the modeling process. The result is a relation that describes the object distance from the camera as a function of changes in the size of the marker. The presented modeling process is performed only once.

The proposed technique of image defuzzification is strong enough to work also for images without standardizing of the brightness, Fig.5. In the study only one change was made to correct the operation of the sensor - image binarization threshold $\Delta \mathrm{T}$ has been lowered from 70 to $50 \pm 3$.

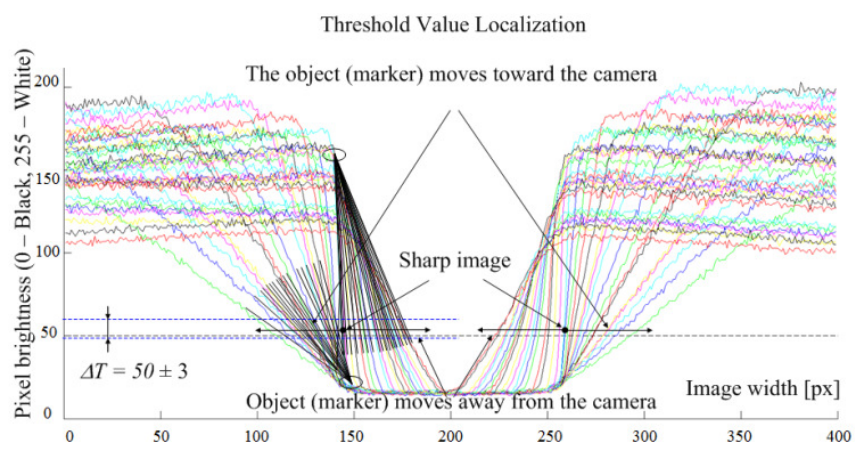

Fig.5. Horizontal line profiles for images from Fig.2.a), Fig.2.c), Fig.2.e) determined by the center of gravity of the object without standardizing brightness.

\section{CONSTRUCTION OF THE VIDEO-MANOMETER}

The active element in the constructed video-manometer was a white silicone flat diaphragm with a thickness of $0.5 \mathrm{~mm}$, Fig.6.a), Fig.6.b). The diaphragm was placed in a modified $2 \mathrm{~N} 25$ solenoid valve. A black circular marker with a diameter of $6 \mathrm{~mm}$ was pasted in the central part of the diaphragm, Fig.6. The marker was monitored by a single board camera with a mounted visible light filter and a lens with a fixed focal length of $f=12 \mathrm{~mm}$. The distance from the front of the lens to the surface of the diaphragm was 60 $\mathrm{mm}$. The camera in the constructed model operated in $I R$ mode with a resolution of $640 \times 480$ pixels at 60 frames per second (fps).

The marker was illuminated with IR LED diodes with a wavelength of $\lambda=850 \mathrm{~nm}$, where the $\mathrm{I}_{\mathrm{F}}$ current was stabilized using the PCA 9622DR chip. The parameters of the IR LEDs were measured by the technique described in [14]. The correlation between the measured width of the marker and the measured gas $\left(\mathrm{CO}_{2}\right)$ pressure was used for pressure measurement. The applied pressure changes the distance of the marker to the front of the camera lens. As a result, the focus in the images becomes blurred. The image underwent segmentation by using digital image processing

techniques. As the result of image processing the view of the marker was isolated, the width of which constitutes the basis for determining the value of the applied pressure.

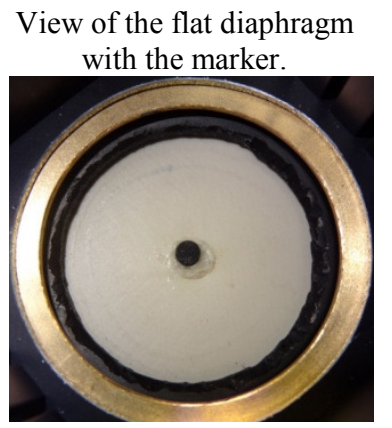

Pixel brightness distribution.

Fig.6.a). View of the flat diaphragm with marker as well as the pixel brightness distribution for flat diaphragm.

View of the convex diaphragm with the marker

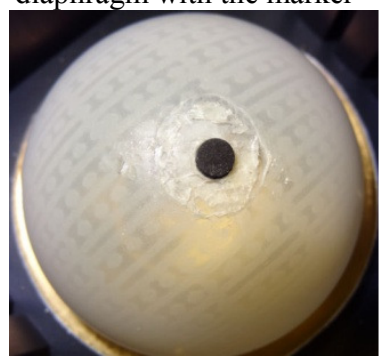
Pixel brightness distribution

Fig.6.b). View of the convex diaphragm with marker as well as the pixel brightness distribution for flat diaphragm.

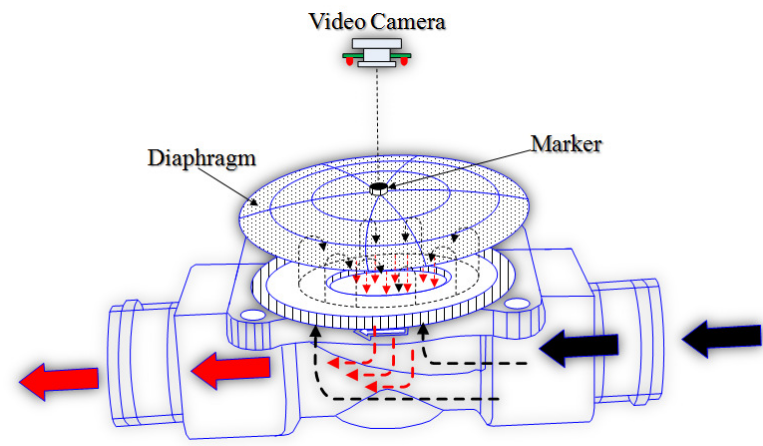

Fig.7. The scheme of the built video-manometer.

The $W$ marker width varies according to the principle: pressure increases $\rightarrow$ blurry image, $W$ increases, Fig.2.b) and Fig.2.a); pressure without changes $\rightarrow$ sharp image, $W$ does not change, Fig.2.c); pressure decreases (underpressure) $\rightarrow$ image blurred, $W$ decreases, Fig.2.d) and Fig.2.e). The measurement of the width of the marker was performed using the technique presented in point 3 . The operation of the developed sensor was examined by measuring the $\mathrm{CO}_{2}$ pressure in the range of $0 \mathrm{mbar}$ to 100 mbar. The model used a flat diaphragm. As a result, considerable deformations of the diaphragm shape were observed, Fig.6.b). Ultimately, the average $\mathrm{CO}_{2}$ pressure measurement error was $1.718 \%$. 


\section{MEASUREMENT SYSTEM CONFIGURATION, VIDEO- MANOMETER CALIBRATION PROCEDURE}

The developed video-manometer was tested at the measuring station shown in Fig.8. The symbol of videomanometer shown in Fig.8. was proposed by the author. The measuring station is equipped with a pressure manometer with a range of 100 mbar and class 1.6 as well as manually closed valves A-D, where: A-intake valve, $\mathrm{D}$ - outlet valve. Valve $\mathrm{C}$ is mounted in front of the calibration manometer. It is used to disconnect the manometer, when the video-manometer is tested in conditions of underpressure.

a)

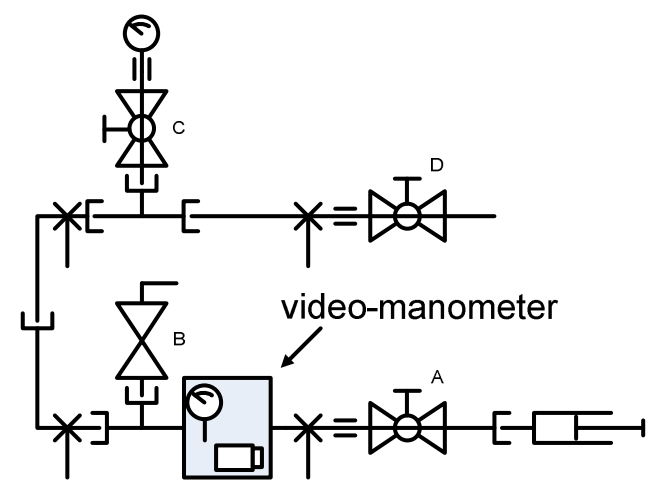

b)

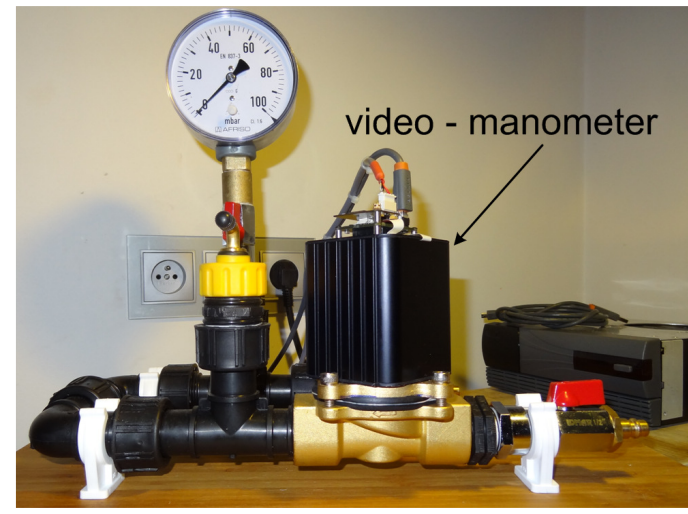

Fig.8. The diagram a) and the view of the measuring station b) for calibrating and testing the video-manometer. The symbol of videomanometer was proposed by the author.

Valves A and D were most commonly used in the experiments. Valve A was used to supply $\mathrm{CO}_{2}$ and to determine the pressure value. Calibration and pressure measurement was performed with valve $\mathrm{D}$ closed. The calibration procedure consisted in forcing pressure values in the range from 0 mbar to 100 mbar in steps of 2 mbar. For each pressure setting 1000 measurements of the marker width were made, from which the average, minimum, and maximum was calculated. The relation between exerted $\mathrm{CO}_{2}$ pressure values and average changes $\mathrm{X}_{\mathrm{S}}$ in the width of the marker is shown in Table 1. Table 1 presents the shortened form of the final results table. During experiments the Lagrange interpolation (LI) and Cubic Spline interpolation (CSI) parameters were determined for the average values of the width of the observed marker.
Table 1. The relation between exerted $\mathrm{CO}_{2}$ pressure values and relative changes in the width of the marker.

\begin{tabular}{|c|c|c|c|c|c|c|}
\hline $\begin{array}{l}\text { Set } \mathrm{CO}_{2} \\
\text { pressure }\end{array}$ & \multicolumn{6}{|c|}{$\begin{array}{l}\text { The relative change in the width of the marker } \\
\text { observed during calibration }\end{array}$} \\
\hline$P_{R}$ & $\mathrm{X}_{\mathrm{MIN}}$ & $\mathrm{X}_{\mathrm{S}}$ & $X_{\text {MAX }}$ & \multicolumn{3}{|c|}{$\mathrm{X}_{\mathrm{S}} \pm \Delta \mathrm{X}_{\mathrm{S}}$} \\
\hline [mbar] & --- & --- & --- & \multicolumn{3}{|c|}{--- } \\
\hline 100 & 1.77 & 1.78 & 1.80 & 1.78 & \pm & 0.02 \\
\hline 96 & 1.74 & 1.75 & 1.77 & 1.75 & \pm & 0.02 \\
\hline 92 & 1.71 & 1.72 & 1.74 & 1.72 & \pm & 0.02 \\
\hline 88 & 1.63 & 1.65 & 1.66 & 1.65 & \pm & 0.02 \\
\hline 84 & 1.58 & 1.59 & 1.60 & 1.59 & \pm & 0.01 \\
\hline 80 & 1.51 & 1.52 & 1.54 & 1.53 & \pm & 0.02 \\
\hline 76 & 1.42 & 1.43 & 1.44 & 1.43 & \pm & 0.01 \\
\hline 72 & 1.37 & 1.38 & 1.39 & 1.38 & \pm & 0.01 \\
\hline 68 & 1.33 & 1.34 & 1.35 & 1.34 & \pm & 0.01 \\
\hline 64 & 1.30 & 1.30 & 1.31 & 1.30 & \pm & 0.01 \\
\hline 60 & 1.27 & 1.28 & 1.29 & 1.28 & \pm & 0.01 \\
\hline 56 & 1.24 & 1.25 & 1.27 & 1.25 & \pm & 0.02 \\
\hline 52 & 1.22 & 1.23 & 1.24 & 1.23 & \pm & 0.01 \\
\hline 48 & 1.20 & 1.21 & 1.22 & 1.21 & \pm & 0.01 \\
\hline 44 & 1.19 & 1.20 & 1.20 & 1.20 & \pm & 0.01 \\
\hline 40 & 1.17 & 1.18 & 1.19 & 1.18 & \pm & 0.01 \\
\hline 36 & 1.16 & 1.17 & 1.17 & 1.17 & \pm & 0.01 \\
\hline 32 & 1.14 & 1.16 & 1.16 & 1.16 & \pm & 0.02 \\
\hline 28 & 1.13 & 1.14 & 1.15 & 1.14 & \pm & 0.01 \\
\hline 24 & 1.12 & 1.12 & 1.14 & 1.12 & \pm & 0.02 \\
\hline 20 & 1.10 & 1.11 & 1.11 & 1.11 & \pm & 0.01 \\
\hline 16 & 1.08 & 1.09 & 1.10 & 1.09 & \pm & 0.01 \\
\hline 12 & 1.07 & 1.07 & 1.08 & 1.07 & \pm & 0.01 \\
\hline 8 & 1.04 & 1.05 & 1.06 & 1.05 & \pm & 0.01 \\
\hline 4 & 1.02 & 1.03 & 1.03 & 1.03 & \pm & 0.01 \\
\hline 0 & 0.99 & 1.00 & 1.01 & 1.00 & \pm & 0.01 \\
\hline
\end{tabular}

The values of exerted pressure were recreated on their basis for the obtained average values of the width of the marker. The calculated $\mathrm{CO}_{2}$ pressure is shown in Table 2. and Table 3. As in the case of Table 1., Table 2. and Table 3. present the shortened form of results tables. The estimated dispersion of pressure measuring results made using the described method is illustrated in Fig.9. The dispersion of the determined pressure values is due to random errors that occur during the measurement of the width of the marker.

The average measurement error equaled 0.396 mbar. The maximum $\mathrm{CO}_{2}$ pressure measurement error during calibration was equal to 1.6 mbar. 


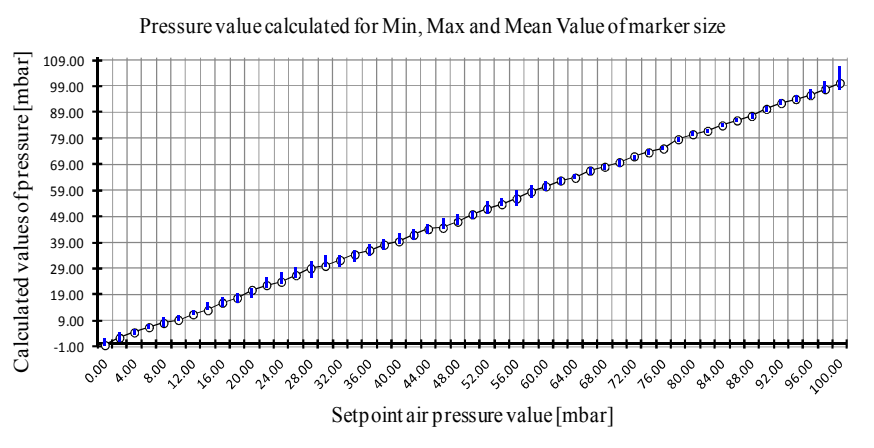

Fig.9. The dispersion of the measured pressure obtained during calibration.

\section{RESEARCH RESULTS}

Video-manometer was tested in the arrangement as presented in Fig.8. The $\mathrm{CO}_{2}$ pressure values were applied in steps of 2 mbar in the range of 0 mbar to 100 mbar. A total of ten series of measurements were made, 50 pressure settings each. Measurement relative error $\delta$ and absolute error were determined for each series. Example results (shortened form) of the measurement of $\mathrm{CO}_{2}$ pressure received during a single experiment are included in Table 2. and Table 3.

Measurement errors were assessed separately for the LI $\delta \mathrm{W}$ as well as the CSI - $\delta \mathrm{S}$. The obtained average error values for the applied $\mathrm{CO}_{2}$ pressure settings are presented in Fig.10. Higher error values were obtained in the experiment for the Lagrange interpolation. The average absolute measurement error in the study for the entire measurement range equaled 0.634 mbar. In comparison, this error for the Cubic Spline interpolation was at a level of 0.562 mbar. The maximum pressure measurement error values in the entire range of measurement stood at 1.972 mbar for the LI and 1.952 mbar for the CSI.

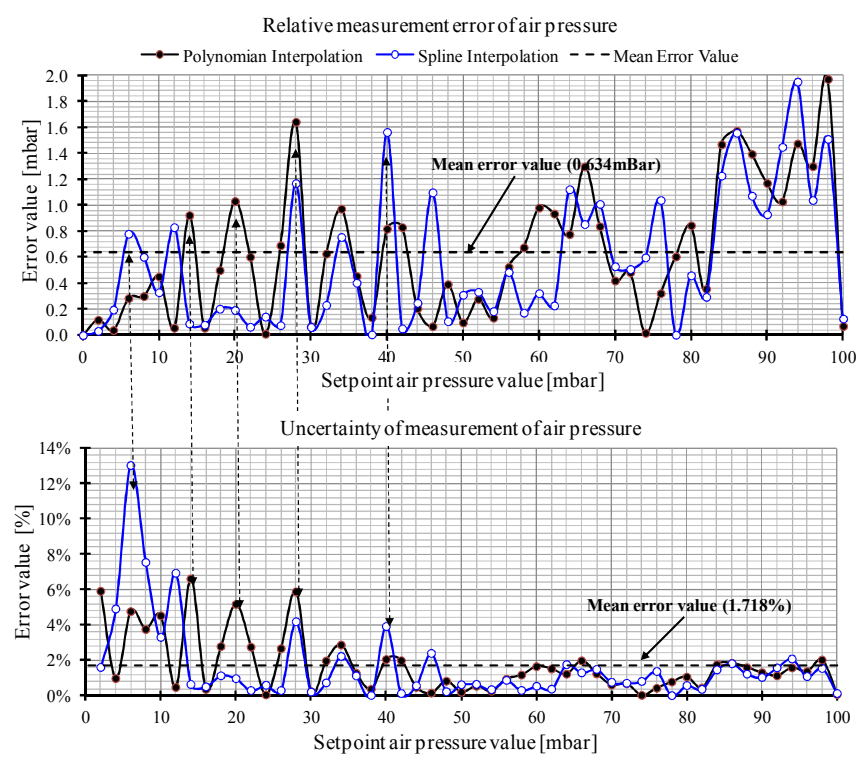

Fig.10. Average absolute and proportional measurement error.
These errors result from a worse adjustment of the model based on the LI for the pressure values of 98 mbar and 94 mbar for the CSI. After limiting the range to 90 mbar the absolute maximum measurement error equaled 1.6 mbar. In terms of percentage, the average measurement error within the entire measurement range equaled $1.718 \%$.

Table 2. Results of the measurement of $\mathrm{CO}_{2}$ pressure received during a single experiment with the use of LI..

\begin{tabular}{|c|c|c|c|c|}
\hline $\begin{array}{l}\text { Set } \mathrm{CO}_{2} \\
\text { pressure }\end{array}$ & \multicolumn{2}{|c|}{$\begin{array}{l}\text { The pressure value } \\
\text { determined for } X_{S}\end{array}$} & \multicolumn{2}{|c|}{$\begin{array}{c}\text { Lagrange } \\
\text { interpolation (LI) }\end{array}$} \\
\hline$P_{R}$ & $P_{\mathrm{M}}$ & $\delta_{\mathrm{P}}$ & $P_{\mathrm{W}}$ & $\delta_{\mathrm{W}}$ \\
\hline [mbar] & [mbar] & --- & [mbar] & --- \\
\hline 100 & 100.23 & $0.23 \%$ & 100.07 & $0.07 \%$ \\
\hline 96 & 95.52 & $0.50 \%$ & 94.70 & $1.35 \%$ \\
\hline 92 & 92.48 & $0.52 \%$ & 90.97 & $1.12 \%$ \\
\hline 88 & 87.52 & $0.55 \%$ & 86.60 & $1.59 \%$ \\
\hline 84 & 83.94 & $0.07 \%$ & 82.53 & $1.75 \%$ \\
\hline 80 & 80.54 & $0.68 \%$ & 80.84 & $1.05 \%$ \\
\hline 76 & 75.04 & $1.26 \%$ & 76.32 & $0.42 \%$ \\
\hline 72 & 72.04 & $0.06 \%$ & 72.48 & $0.67 \%$ \\
\hline 68 & 68.06 & $0.09 \%$ & 68.83 & $1.22 \%$ \\
\hline 64 & 63.83 & $0.27 \%$ & 64.77 & $1.20 \%$ \\
\hline 60 & 60.54 & $0.90 \%$ & 60.98 & $1.63 \%$ \\
\hline 56 & 55.86 & $0.25 \%$ & 56.52 & $0.93 \%$ \\
\hline 52 & 51.96 & $0.08 \%$ & 52.27 & $0.52 \%$ \\
\hline 48 & 47.00 & $2.08 \%$ & 47.60 & $0.83 \%$ \\
\hline 44 & 44.14 & $0.32 \%$ & 44.20 & $0.45 \%$ \\
\hline 40 & 39.44 & $1.40 \%$ & 40.81 & $2.03 \%$ \\
\hline 36 & 36.02 & $0.06 \%$ & 36.45 & $1.25 \%$ \\
\hline 32 & 32.28 & $0.88 \%$ & 32.62 & $1.94 \%$ \\
\hline 28 & 29.10 & $3.93 \%$ & 29.64 & $5.86 \%$ \\
\hline 24 & 23.90 & $0.42 \%$ & 24.01 & $0.04 \%$ \\
\hline 20 & 20.78 & $3.90 \%$ & 21.03 & $5.15 \%$ \\
\hline 16 & 15.85 & $0.94 \%$ & 15.94 & $0.38 \%$ \\
\hline 12 & 11.41 & $4.92 \%$ & 12.05 & $0.42 \%$ \\
\hline 8 & 8.09 & $1.13 \%$ & 7.70 & $3.75 \%$ \\
\hline 4 & 4.47 & $11.75 \%$ & 3.96 & $1.00 \%$ \\
\hline 0 & -0.41 & --- & 0.00 & --- \\
\hline
\end{tabular}


Table 3. Results of the measurement of $\mathrm{CO}_{2}$ pressure received during a single experiment with the use of CSI..

\begin{tabular}{|c|c|c|c|c|}
\hline $\begin{array}{l}\text { Set } \mathrm{CO}_{2} \\
\text { pressure }\end{array}$ & \multicolumn{2}{|c|}{$\begin{array}{l}\text { The pressure value } \\
\text { determined for } \mathrm{X}_{\mathrm{S}}\end{array}$} & \multicolumn{2}{|c|}{$\begin{array}{c}\text { Cubic Spline } \\
\text { interpolation (CSI) }\end{array}$} \\
\hline$P_{R}$ & $P_{\mathrm{M}}$ & $\delta_{\mathrm{P}}$ & $P_{\mathrm{S}}$ & $\delta_{\mathrm{s} .}$ \\
\hline [mbar] & [mbar] & --- & [mbar] & --- \\
\hline 100 & 100.23 & $0.23 \%$ & 99.87 & $0.13 \%$ \\
\hline 96 & 95.52 & $0.50 \%$ & 94.96 & $1.08 \%$ \\
\hline 92 & 92.48 & $0.52 \%$ & 90.55 & $1.58 \%$ \\
\hline 88 & 87.52 & $0.55 \%$ & 86.92 & $1.23 \%$ \\
\hline 84 & 83.94 & $0.07 \%$ & 82.77 & $1.46 \%$ \\
\hline 80 & 80.54 & $0.68 \%$ & 80.46 & $0.57 \%$ \\
\hline 76 & 75.04 & $1.26 \%$ & 77.04 & $1.37 \%$ \\
\hline 72 & 72.04 & $0.06 \%$ & 72.50 & $0.69 \%$ \\
\hline 68 & 68.06 & $0.09 \%$ & 69.01 & $1.49 \%$ \\
\hline 64 & 63.83 & $0.27 \%$ & 65.12 & $1.75 \%$ \\
\hline 60 & 60.54 & $0.90 \%$ & 60.32 & $0.53 \%$ \\
\hline 56 & 55.86 & $0.25 \%$ & 56.48 & $0.86 \%$ \\
\hline 52 & 51.96 & $0.08 \%$ & 52.33 & $0.63 \%$ \\
\hline 48 & 47.00 & $2.08 \%$ & 48.10 & $0.21 \%$ \\
\hline 44 & 44.14 & $0.32 \%$ & 44.24 & $0.55 \%$ \\
\hline 40 & 39.44 & $1.40 \%$ & 41.56 & $3.90 \%$ \\
\hline 36 & 36.02 & $0.06 \%$ & 36.40 & $1.11 \%$ \\
\hline 32 & 32.28 & $0.88 \%$ & 32.23 & $0.72 \%$ \\
\hline 28 & 29.10 & $3.93 \%$ & 29.17 & $4.18 \%$ \\
\hline 24 & 23.90 & $0.42 \%$ & 24.14 & $0.58 \%$ \\
\hline 20 & 20.78 & $3.90 \%$ & 20.19 & $0.95 \%$ \\
\hline 16 & 15.85 & $0.94 \%$ & 16.08 & $0.50 \%$ \\
\hline 12 & 11.41 & $4.92 \%$ & 12.83 & $6.92 \%$ \\
\hline 8 & 8.09 & $1.13 \%$ & 7.39 & $7.63 \%$ \\
\hline 4 & 4.47 & $11.75 \%$ & 3.80 & $5.00 \%$ \\
\hline 0 & -0.41 & --- & 0.00 & --- \\
\hline
\end{tabular}

\section{CONCLUSiOnS}

The paper presents the design and research results of the developed and practically built video-manometer. The constructed video-manometer using a flat silicone diaphragm in the full measuring range, i.e. from 0 mbar to $100 \mathrm{mbar}$, is a class 2 . After limiting the scope to the value of 90 mbar the device has become class 1.6.

The proposed pressure sensor has the following properties: non-contact measurement; galvanic isolation between the sensor and the increased and/or decreased pressure chamber; high sensitivity, reproducibility and accuracy of carried out measurements; interference resistance; lack of frictional and mechanical components; design simplicity; small and scalable sensor sizes; the ability to measure the pressure of gases using diaphragms: flat, corrugated, bellows and capsulated; low-cost implementation; the possibility of using video fiber optics; real-time visual sensor operation self-diagnostics, virtually any number of defined alarm thresholds and methods of informing on their occurrence, digital output.

Application of the developed technique for measuring the width of the marker in conditions where the occurrence of motion blur takes place and video sensor, helped create a unique manometer (video-manometer) with a possible visional self-diagnostics feature executed in real-time. Selfdiagnosis could be easily carried out by analyzing the changes in the image of the measuring diaphragm obtained from the interior of the video-manometer.

The developed measurement technique in sensory applications provides features unavailable for other types of transducers. It can be successfully used to build videotransmitters for other physical quantities such as distance, liquid level, temperature, etc. The presented measurement technique allows monitoring movements of the flat and corrugated diaphragm. The signal obtained directly from the sensor, without the need to convert the measured physical quantity, can be used to determine the signal error in video feedback of control systems.

Presented measurement technique is the subject of a patent pending P.408076.

\section{ACKNOWLEDGMENT}

The author would like to thank Professor Tadeusz Pustelny as well as Grzegorz Konieczny PhD. Eng. from the Silesian University of Technology, whose publications have been an inspiration to this work. The work was originally presented at the 8th Conference Integrated Optics - Sensors, Sensing Structures and Methods, IOS'2014 sponsored by the Polish Academy of Sciences.

\section{REFERENCES}

[1] Torres, J.A., Velazquez, G. (2005). Commercial opportunities and research challenges in the high pressure processing of foods. Journal of Food Engineering, 67 (1-2), 95-112.

[2] WIKA Instrument, LP. (2015). Is a pressure gauge important to everyday operations? http://www.wika.us/.

[3] Abruss, A., Guo, Y., Wang, T., Gu, F.S., Ball, A.D., Brown, D. (2015). Investigation of the effect of biodiesel blends on fuel injection pumps based on vibration and pressure measurements. Mechanisms and Machine Science, 23, 535-546.

[4] Haering, E.H. Jr. (1995). Airdata measurement and calibration. NASA Technical Memorandum 104316. National Aeronautics and Space Administration, Washington, DC.

[5] Konieczny, G., Opilski, Z., Pustelny, T., Maciak, E. (2009). State of the work diagram of the artificial heart. Acta Physica Polonica A, 116, 344-347. 
[6] Sismanoglu, B.N., Grigorov, K.G., Caetano, R., Rezende, M.V.O., Hoyer, Y.D. (2010). Spectroscopic measurements and electrical diagnostics of microhollow cathode discharges in argon flow at atmospheric pressure. European Physical Journal D, 60 (3), 505-516.

[7] Beckerath, A., Eberlein, A., Hermann, J., Kersten, P., Kreutzer, J. (2008). WIKA Handbook: Pressure and Temperature Measurement. U.S. Edition. WIKA Instrument Corp.

[8] Webster, J.G. (ed.) (1999). The Measurement, Instrumentation and Sensors: Handbook. CRC Press.

[9] Land, H.B. III, Eddins, C.L. (2004). Optical pressure measurement: Using fiber optic transducers in hypersonic flight vehicles. IEEE Instrumentation \& Measurement Magazine, 7 (3), 38-45.

[10] Pinet, É. (2011). Pressure measurement with fiber-optic sensors: Commercial technologies and applications. In 21st International Conference on Optical Fiber Sensors, Proc. SPIE 7753.

[11] Favero, I., Karrai, K. (2009). Optomechanics of deformable optical cavities. Nature Photonics, 3, 201205.

[12] Vaughan, J.M. (1989). The Fabry-Perot Interferometer: History, Theory, Practice and Applications. CRC Press, 89-132.

[13] Urban, F., Kadlec, J., Vlach, R., Kuchta, R. (2010). Design of a pressure sensor based on optical fiber Bragg grating lateral deformation. Sensors, 10, 1121211225.

[14] Życzkowski, M., Szustakowski, M., Rożanowski, K., Murawski, K., Karol, M., Markowski, P. (2012). research and parameter optimization of the infrared sensor for eye track. Acta Physica Polonica A, 122, 942-945.

Received November 19, 2014 Accepted June 24, 2015. 